\title{
Forensic textile damage analysis: recent advances
}

This article was published in the following Dove Press journal:

Research and Reports in Forensic Medical Science

\section{Graham A Williams \\ Department of Criminal Justice and Forensic Science, School of Law, Policing, and Forensics, Staffordshire University, Stoke-on-Trent, \\ Staffordshire, UK}

Correspondence: Graham A Williams Department of Criminal Justice and Forensic Science, School of Law, Policing, and Forensics, Staffordshire University, Leek Road, Stoke-on-Trent, Staffordshire ST4 2DF, UK

Email graham.williams@staffs.ac.uk

\begin{abstract}
Textiles damage analysis is an invaluable tool in forensic investigations and can be used to help resolve cases of stabbings, shootings, and sexual assaults. It also has the potential to resolve arson attacks and "acid attacks". Here, the literature is explored in order to review research relating to blunt force damage, stab and slash cuts, the phenomenon of falsification of evidence in sexual assaults, projectiles damage, and the effect of decomposition upon textiles damage, among others. It is clear that there is a paucity of research in the area, but where the research has been carried out, it has been informative and impactful. However, there are areas that need further work, including a better understanding of normal wear and tear, and the potential for chemicals to cause textiles damage. One area that is in a significant need of further work is the development of a robust interpretational framework.
\end{abstract}

Keywords: forensic textile damage, clothing damage analysis, fabric damage, stab cuts, decomposition, interpretation, sexual assaults

\section{Introduction}

Forensic textiles damage is a niche area of the forensic sciences, and it is where the damage to fabric and textiles is analyzed with a view to providing information to the forensic investigation. It has been utilized in stabbing cases where the damage can be examined in order to provide an opinion as to the implement and actions that have caused such damage. In such cases, clothing damage can be thought of as a two-dimensional version of the three-dimensional wound. While a wound can provide further information, this is not always available - due to medical intervention (either by stitching the wound up or by cutting into it further to repair underlying damage) or where the deceased has undergone decomposition. In such cases, the clothing may then be analyzed. This discipline can also be used in sexual assaults, where clothing has been subjected to a blunt force in that the clothing or underwear has been pulled off with a degree of force, emphasized by the complainant also pulling on the garment.

There are also efforts to understand the impact of projectiles causing damage to clothing - not just from bullets but also from air pellets, arrows, and crossbow bolts.

More recently, with increasingly high-profile "acid attacks" being committed where corrosive substances are being thrown upon individuals and items (such as vehicles), there is an effort being undertaken to identify chemical damage to clothing in an effort to provide some evidence linking the suspect to a corrosive substance. 
Consequently, the area of forensic textiles damage (or clothing damage analysis) is a specialized one that currently has great use in progressing criminal investigations, and with further research, it could be even more useful.

\section{Blunt force damage}

Blunt force damage is typically characterized by tearing/ ripping and punching/kicking and is generally a catch-all phrase for damage not caused by a sharp implement. This occurs when textiles or clothing are placed under strain, for example, by being grabbed and pulled, until the fabric tears. Such tears primarily occur at the weakest part of the garment, which is usually the seam. Blunt force impact (BFI), such as from punching or any forceful contact from an object, may also lead to damage of the textiles, such as distortion or widening of the warp and weft.

Recent research in this area includes a study by Daroux et al, ${ }^{1}$ which explores the effect of laundering on BFI on fabrics. The authors noted that the majority of research in textiles damage focuses mainly on stab cuts and there is very little on BFI other than an examination of the associated injuries. They noted that BFI can have a high kinetic energy and how this energy is transferred to the fabric affects the subsequent damage morphology. Much of the research in this area is focused on improving ballistic clothing (ie, "bulletproof vests"). Such kinetic energy being transferred can result in stretching and distortion of individual yarns and cause such yarns to rub against each other, potentially being damaged in the process. Many factors affect this, such as the flexibility and resiliency of the yarns as well as how tightly the yarns are packed during the weaving process. Needless to say, many variables associated with the blunt instrument as well as the nature of the action will have a widely varying effect on the damage appearance. However, typically the authors reported that such damage mainly consists of yarn flattening, fiber fractures, and holes.

On examining the damage prior to laundering, the authors noticed that BFI damage could be observed, but interestingly noticed that holes only appeared if the BFI strength was sufficiently high and postulated that the absence of holes could indicate a low BFI and potentially provide information regarding the alleged incident.

Exploring the effect of laundering, the authors indicated that fabrics being laundered prior to the BFI did not have an effect on the appearance; thus, the laundering did not weaken the textile to the extent that it became easier to damage, despite the authors observing damage caused by a single laundry cycle. Interestingly, the authors also postulated that this method could be used to provide an opinion on whether the garment is new or used.

The authors indicated that laundering actually helped remove some visible damage, particularly the distortions caused by BFI, that is, the swelling and twisting of the fabrics, to the extent that the damage could not be observed macroscopically. However, the microscopic damage was still visible.

A study by Geisenberger et $\mathrm{al}^{2}$ explored the influence of BFI or blunt trauma in deceased individuals. The authors report that many deaths are caused by blunt trauma, such as being hit by a vehicle. The authors present six case studies where the deceased suffered blunt trauma due to being hit by vehicles, including trains. In all cases, the authors observed an intense yellow discoloration of the clothing that could be correlated with an area of intense contusion. The clothing was light-colored. The authors hypothesized that this yellow staining was a result of fat coming out of contused adipose tissue. In order to verify this, the authors conducted gas chromatography-mass spectrometry analysis and compared the stained sample with reference adipose tissue from the deceased, which supported their hypothesis that the yellow staining on the clothes was from fat. The authors noted that all of the deceased in this study were either overweight or obese. The authors accepted that the absence of underweight victims could be detrimental to the validity of the study.

In all cases, the area of contusion also had lacerations, thus presenting an exit route for the contused adipose tissue. Obviously, where there are lacerations a significant amount of blood may also be expected; thus, it is possible for such discolorations to be masked. However, Geisenberger et al did not explore whether these yellow stains could be an alternative substance, such as diluted/mixed blood or even urine stains.

While these stains do not technically count as damage, the interaction between body fluids and the garment can affect the analysts' observations. For example, the presence of blood on stab cuts may have a preservative effect on the morphology of the cut fabrics. This can also be applied to semen stains on underwear where they have been pulled off prior to the rape and then replaced following the incident. The presence of semen on the area of damage may well have an effect on any damage to the underwear.

\section{Sharp force damage}

Sharp force damage is often referred to as stab cuts, whereby a sharp implement has penetrated through a piece of fabric, thereby leaving a hole that reflects the cutting implement itself. For example, a single-edged blade (such as a kitchen 
knife) will leave a different-shaped cut to one created by a double-edged blade (diving or fishing knives). A blade with a serrated edge (such as a bread knife) will leave yet a different-shaped hole.

Not only does the implement itself affect the shape and size of the cut, but the action can have a big effect. For example, a stab cut is generally created by thrusting a sharp implement into the fabric tip first with the damage being caused as the fabric runs along the blade. However, if the sharp implement is swung across the surface of the fabric, then a slash cut is generated by the swinging motion.

There have been some efforts in trying to correlate the appearance of stab cuts with features of the cutting implement, the actions used, and the fabric itself. For example, Nic Daéid et $\mathrm{al}^{3}$ explored whether stab cuts could be correlated with the wounds themselves, although the purpose of this was unclear. The authors demonstrated that if the fabric was loose against the skin, then the length of the stab cuts and the length of the wounds were not significantly different, whereas if the fabric was stretched, then the lengths were significantly different due to the distortion of the fabric. This highlighted how important it is for the damage analyst to be aware of how the clothing was worn and whether it was a loose fit or a tight fit.

Kemp et $\mathrm{al}^{4}$ conducted a reasonably comprehensive study exploring the relationship between weapons and the damage created making a number of observations. Primarily, that differentiation of blades was possible by observing variances in size and morphology of the cuts. This includes directionality of the cuts if there were variations along the cutting edge, that is, sharpness, shape, or cutting gradient. The authors also observed that the textiles themselves had a significant effect on the stab cuts. They examined the stab cuts at varying levels, including from single threads, and concluded that it was not possible, or at least safe, to offer an opinion as to what caused the damage. While most of these findings were already known, this study provided a very useful clarification of the issues associated with stab cut examination; this study did provide a very useful insight, and that is the comparison of impact rigs vs human participant trials. The authors stated that they were able to find better correlations when they removed the complex variables associated with a human creating the stab cuts compared to the use of an impact rig.

There have been a number of "meta-studies" where researchers have evaluated an effective way to create and record various damages to textiles. In 2004, Jussila ${ }^{5}$ provided a useful review and proposed a standard method for preparing ballistic gelatine. This goes some way to allowing research produced by different groups to be compared in a meaningful way. However, this does not appear to be taken up with a further study produced by Carr and Wainwright ${ }^{6}$ which explores the variability of simulants used. The authors explored 20\% gelatine, expanded polystyrene (EPS), and deboned rolled pork shoulder joint with skin. The authors recommended the use of EPS as it gave the least variabilities when recreating stab cuts and was the easiest and quickest to prepare. The authors acknowledged that this would not replicate human tissue. Thus, the use of the back simulants largely depends on the objective of the study. For example, if the intention is to understand the stab dynamics and the variables accounting for this, then the use of EPS would be best. If, however, the study is more about the appearance of the damage in stabbings, then an alternative simulant may be more appropriate.

Interestingly, as part of this study, Carr and Wainwright developed an impact rig to explore this. An impact rig is a method by which stab cuts or damages can be created in a programmable way and various factors recorded - typically the forces involved in creating such cuts. This is something that has also been looked at by Benson et al, ${ }^{7}$ who developed a "stabbing machine".

Although a stab cut is often used as a generic term for any cut made with a sharp implement, it does refer to a penetrative thrust with a sharp implement. Thus, there is also a slash cut, which is caused by a swinging action with a sharp implement. Anecdotally, this may be more common than a stab cut. However, there has been even less research conducted in this area. One such study by Bostock et $\mathrm{al}^{19}$ explored slash cuts and the correlation between the sharpness of an implement and its corresponding damage. Interestingly, the authors presented two novel techniques, one for measuring the sharpness of the knife without blunting it (relative sharpness index) and proposed a categorization of such damage. The authors were able to demonstrate a correlation between the sharpness and the damage caused.

\section{Projectiles damage}

This is the damage caused by any object that is projected through the air and typically intending to penetrate the body. The most obvious example is that of bullets discharged from firearms, but it also includes air pellets, arrows, and crossbow bolts.

There is a good body of work relating to the interaction between a projectile and a wound, including the evaluation of various stimulants and ballistic gels as an effective model for research. ${ }^{5,6}$ This body of research does also include the 
observation that textile fibers can be found within the wound tract, where the projectile passed through a textile layer before penetrating the body. ${ }^{9}$ However, there is little recent published work upon the impact of projectiles on the clothing itself, with the bulk of the work exploring the impact that clothing has on wound appearance and morphology.

Wightman et $\mathrm{al}^{10}$ explored the interaction between air weapon pellets and the clothing. Although much of this work is more about the effect that clothing has on the subsequent injury (or damage caused to the ballistic gel), it did briefly explore the impact of the air pellet on the clothing itself. One observation was that clothing was capable of acting as a barrier to air pellets. However, this is contingent on a complex array of factors, such as the distance between weapon and target and the nature of the fabric itself. The authors demonstrated that most fabrics will stop an air pellet when fired from a distance of $18.2 \mathrm{~m}$ and that denim will stop an air pellet from $9.1 \mathrm{~m}$ whereas cotton fabrics will not. They did not comment on any damage that may have been present on the textile.

Research by MacPhee et $\mathrm{al}^{11}$ explored the influence of clothing on damage caused by different types of arrowheads. It highlighted a number of cases where bow and arrows and crossbows were used to cause death, including a case of suicide by crossbow, among others. ${ }^{12-14}$ This study indicated a number of different shapes of arrowheads, including "bullet", "blunt", "judo", and "broadhead". Each of these had varying sizes and cross-sectional surface areas. In addition, this study explored the impact of loose clothing vs tight clothing. The clothing explored were jeans (of different cotton compositions) and a t-shirt with $95 \%$ cotton. The clothing was placed onto a gelatine block, and the depth of penetration recorded along with observations of the damage itself. In general, it demonstrated that the presence of clothing, somewhat unsurprisingly, reduced the penetrative ability of the projectiles. It was the broadhead arrow that caused the most damage as it had the presence of blades on the head and the widest cross-sectional area.

Just as with the study by Wightman et al, the study by MacPhee et al focused more upon the influence of the clothing to the damage or injury itself, but it also included reference to the clothing damage and highlighted that the cross-sectional area and morphology had a significant impact on the clothing damage. For example, the broadhead arrowhead had three blades emerging from the central shaft at the tip and damage caused by this actually showed a central hole with three cuts emerging from it - which is very distinctive. MacPhee et al observed that despite the size of the projectile remaining the same, the nature of the fabric itself caused variations; for example, a tight-fitted clothing gave a larger dimension than loose-fitted clothing, with the authors explaining that this would be a result of the fabric being stretched at the time of being damaged, thus creating a larger hole.

The authors also noted that damage caused to fabrics by the "judo" arrowhead was minimal. The "judo" arrowhead has four spring-loaded arms that are deployed in flight and is designed to catch on grass or tree stumps to prevent them from being lost. The authors stated that the damage was difficult to observe as the damage looked like normal wear and tear. The image published as part of the study indicated a number of indentations with no penetration.

The authors acknowledged that there were significant variations associated with this study that were not incorporated. For example, the clothes themselves were usual "civilian" clothing, where hunting clothes may offer more protection; only one bow was used for the study, and the influence of different and stronger bows was not included, as well as the potential influence of the archer themselves.

One area of clothing damage that appears to be somewhat neglected is the one of firearms damage with some very limited research being conducted. One recent study by Hinrichs et $\mathrm{al}^{15}$ attempted to estimate shooting distances by characterizing the firearms discharge residue around the bullet holes using scanning electron microscopy (SEM). Estimating firing distance is a key element of shooting reconstructions and generally uses a combination of the bullet hole appearance and firearms discharge residues around the bullet hole itself. Here, the authors proposed using variable pressure SEM-energy dispersive X-ray spectroscopy. Again, while the study was primarily focused on the use of a novel technique for shooting estimations, it also made references to the textile damage itself. The authors noted macroscopic changes in the appearance of the textile, primarily around the appearance and dispersal of "soot" from the firearm. Reviewing the published images showed little variation in the actual damage itself, although the different amounts of distortion between soft contact and from $20 \mathrm{~cm}$ away appear to be substantial.

Following microscopic analysis (using SEM), the authors observed that the damage (tapered ends due to mechanical extension) was similar at various shooting distances in cotton fabrics. However, when examining the synthetic fibers in denim, the authors observed melted fibers where the polyester was exposed to heat above $250^{\circ} \mathrm{C}$ resulting in fused fiber endings. Where there were a cotton weft and a polyester warp, 
this resulted in the warp melting, but the cotton remaining intact, leading to one-directional tearing following a contact shot, as the damage followed the path of least resistance, that is, along the melted warp. This fusing of fibers was still observed at $2.5 \mathrm{~cm}$.

This limited body of research into the effect of projectiles on clothing damage demonstrates that the interaction between the textile and the projectile is highly dynamic and does require a great deal of further study.

\section{Damage in sexual assault cases}

Damage in sexual assault cases is typically blunt force damage, where clothing and underwear have been removed by force - often with the complainant fighting to keep the clothes on. This can result in highly dynamic and complex damage patterns to the clothing. Although by no means definitive, the presence of significant damage to the clothing can be evidence of absence of consent in sexual assault cases.

One area that has been highlighted as a potential issue in the use of damage analysis in sexual assault is the phenomenon of falsification of evidence - either to back up a false allegation or to bolster the perception of weak evidence in a genuine allegation. A study by Williams and Haider ${ }^{16}$ reported a case where a bra was removed with sufficient force to result in the separation of the bra cups and damage to the bra straps. However, the hook and eye fastening were intact. The defense team claimed that since the hook and eye fastening were intact, the damage must have been falsified as it was felt that the hook and eye fastening would be the first bit to be damaged if the bra was removed so forcefully. The authors then undertook a series of recreation studies where a bra was forcefully removed from a volunteer and demonstrated that eight times out of nine the hook and eye fastening remained intact. The authors highlighted the need to have a clothing damage expert involved in such cases. It was noted that if the force was applied from the front, the hook and eye fastening would be lying flush against the skin; if the force was applied directly to the hook and eye fastening, then it is likely that this will be damaged first.

The allegation of deliberate damage or falsification appears to be a relatively common one, with further studies exploring this phenomenon. Daly et $\mathrm{a}^{17}$ reported on two case studies focusing on the hook and eye fastening on the back of a bra. In one case study, the hook and eye fastening on the bra were distorted with the hooks having been bent open - allegedly as a part of the bra being pulled off. However, the authors observed tool marks on the hooks that would have been caused by contact between the surface and an implement. There was no corresponding damage on the eye fastenings. A force analysis of the hooks highlighted that considerable force would have been required to distort the hooks, a greater force than is required to cause damage to the surrounding fabric, but none was observed. Thus, the finding suggested that the hooks had been deliberately straightened.

However, the second case study highlighted a case of consensual but unlawful intercourse (under age of consent) where a bra was recovered with distorted and straightened hooks. It emerged that the bra was an old one and that the girl had manipulated the hooks so she could continue to wear the bra.

This second case study served to highlight the contextual sensitivity surrounding textiles damage analysis as the first case study demonstrated a case of falsified damage, but the same damage was also observed in a genuine consensual case study.

The case studies relating to the phenomenon of falsified or deliberate damage highlight the need for a greater understanding of natural wear and tear of the garment. For example, in the first case study by Williams and Haider, the investigators felt that the bra could not sustain such damage without causing damage to the hook and eye fastening, but a very simple reconstruction experiment showed this to be incorrect. Another case study demonstrated that the hooks could be manipulated, thus making it look like the complainant has falsified the damage, but that same paper highlighted that such damage can also be caused by the wearer trying to fix the bra to make it wearable. This highlights the need for a greater understanding of the natural and innocuous forces that work on textiles. For example, a study by Beever ${ }^{18}$ explored natural weathering, whereby the pieces of fabrics were left exposed to the weather to explore any changes in the fabric. This research was extremely useful in establishing a baseline for natural wear and tear and lays the foundation for further research into this area.

\section{Decomposition}

If the deceased had received a stab cut, for example, and the body is unfound for a period of time, then the integrity of the clothing evidence may be affected by the decomposition. A study by Bostock and Williams ${ }^{8}$ showed that the edges of the stab cuts become more frayed when placed on a source of decomposition, when compared with stab cuts without a source of decomposition. Empirical observations indicated that this may have been caused by insect activity. In the experiment, the material was wrapped around a source of decomposition, with the only entry point being the stab cut. 
Thus, insect would be attracted to the meat and lay eggs which hatched into larvae. These could be observed moving in and out of the package via the stab cut. It appears to be thought that the movement of the larvae exacerbated the fraying of the cut edge. This would have the effect of reduced confidence as to whether or not the damage could be classified as a stab cut rather than another form of damage. One limitation of this study was the absence of any blood on the edges of the cut; in a more realistic situation, this would be expected.

Another study by Ueland et $\mathrm{al}^{20}$ explored the effect of decomposition and burial upon the degradation of shirting fabrics. The purpose of the study was to recreate clandestine burials and the effect that this may have on the integrity of clothing damage evidence. This study built upon previous studies by Lowe et al, ${ }^{21}$ whereby it was demonstrated that the cadaverous remains actually had a preservative effect on textiles when compared to fabrics not wrapped around a source of decomposition. Ueland et $\mathrm{al}^{20}$ were able to replicate this phenomenon and demonstrated again that textiles exposed to decomposition fluids were somewhat preserved.

Mitchell et $\mathrm{al}^{22}$ also looked at the influence of burial upon textiles and explored the influence of two different soil types, clay and sand. Interestingly, the authors did not include a decomposition source within the experimental design, and thus, this study evaluated the influence of the burial conditions on the fabrics alone. They also looked at different fabrics, comparing $100 \%$ cotton vs a cotton/polyester blend, and found that in all cases, the cotton/polyester blend was the most affected with greater staining and less force required to tear it.

A study of this nature is vital to understand the influence of decomposition on textiles, in that it is necessary to understand how textiles change over time in normal conditions - something that is explored well by Beever. ${ }^{18}$

The study by Mitchell et $\mathrm{al}^{22}$ does take on a new perspective when considering the work by Ueland et $\mathrm{al}^{20}$ and Lowe et al, ${ }^{21}$ in that burial conditions do cause textiles to degrade, but if wrapped around a cadaver, then the textiles may well be preserved - which is somewhat paradoxical when considering the study of Bostock and Williams. ${ }^{8}$ However, the key difference is that in the study of Bostock and Williams, ${ }^{8}$ the textiles were not actually buried, but were simply left on the surface or just under the surface, whereas in the other studies, the textiles were buried to a relatively substantial depth.

Compared to other areas of textiles damage research, the interaction between the decomposition and the fabric is one that has been explored in relative greater depth, already throwing up unexpected outcomes, that is, the paradoxical aspect of the decomposing body preserving the textile.

\section{Thermal and chemical damage}

One of the main reasons why textiles damage is such a useful piece of evidence is that most crimes are committed by people wearing clothes to people wearing clothes, so if someone were to commit arson or an "acid attack", then there is potential for the clothing to either receive thermal damage from igniting a fire, for example, holding a Molotov cocktail can result in flames being very close to the sleeve, or receive chemical damage from corrosive substances splashing back on to the offender. "Acid attacks" are becoming more and more high profile, and thus, there is a need to understand the effect that such corrosive substances can have on the clothing. Currently, there appears to have been no published works relating to the influence of corrosive substances upon textiles.

However, there appears to have been some limited work on thermal damage to textiles where Leung and Halliday ${ }^{23}$ explored the impact of flash burning on the textile. Flash burning results where the vapor from a volatile flammable liquid (such as petrol) mixes with the surrounding air creating a cloud, and the ignition of this vapor cloud will result in a flame front that flashes through. Items exposed to this flash front may then be subjected to directional heat damage, such as clothing and/or shoes.

The authors indicate that the extent of this damage can be dependent upon the susceptibility of the fabrics themselves (melting points, thermal capacities, etc), the duration of contact between the items and the flash front, and the amount of flammable vapor present. In most cases, this flash burning is described as transient in that it is a quick event, and therefore, such textile damage could be superficial.

Flash front is also encountered when exposed to detonations or explosions, but it is clear that there is no recent research in this area.

\section{Analysis and interpretation}

One of the weakest areas in textile damage is the framework in which the findings are analyzed and interpreted. A significant criticism of the field is how the damage is identified and categorized and is often referred to as subjective. This is highlighted by the fact that guidance highlights the importance of experience in interpreting the findings.

Attempts at standardizing the terms used have been conducted by Taupin; ${ }^{24-27}$ however, a framework for interpreting is currently absent. 
Typically, most practitioners will use a Bayesian approach where they will evaluate the probability of getting a particular type of damage given two mutually exclusive hypotheses. Prior to $\mathrm{RvT}^{28}$ forensic practitioners would use the benefit of their experience to semi-quantify the probability of obtaining a particular damage, given a particular scenario. However, post-RvT, this approach was not allowed, given the lack of a robust data set, and currently, advice on this matter can be limited to providing an unquantified level of support for one hypothesis over another. In order to address this, robust data sets need to be formulated that allow for the generation of reliable probability values that can be inputted into the case-specific probability model. ${ }^{29}$

However, Boland et $\mathrm{al}^{30}$ have proposed a systematic approach to analyze textiles damage, albeit in a sexual assault context, but it would also be useful in the wider context. This proposes a four-tier system - 1) gross morphology (which is referred to by other authors as "macroscopic examination"), 2) microscopic examination, 3) reconstruction, and 4) assessment and interpretation. Other than the inclusion of the reconstruction step, this model appears to be utilized by other researchers, although not explicitly stated. The authors also highlight the contextual sensitivity of this approach. However, this study did not suggest an interpretational framework.

\section{Challenges in textile damage analysis}

One of the major challenges in textile damage analysis is mainly centered around quality and the lack of consistency across the discipline. Although the works of Taupin ${ }^{24-27}$ have led to a standardization of terminology, the actual application of the science to casework remains somewhat fragmented. In order to rectify this, a clear interpretational procedure needs to be developed that is robust despite the RvT ruling. RvT refers to the court ruling where expert opinion could only be offered when backed by rigorous and relevant databases and that experts could not use their experience to inform their decisions. This ruling has a significant effect on the forensic practitioners ${ }^{28}$ and meant that some evidence could be rendered inadmissible. Although Boland et al made an effort, it still fell short.

Another area is the development of robust data sets which allows for the reliable evaluation of activity-level hypotheses. For example, what is the probability of observing a stab cut given that the victim was stabbed with a single-edged blade? Logic and common sense indicate that this would be very high $(P=0.99)$ potentially even at $P=1$. However, when the basic principle of forensic interpretation comes in to play, the question is then asked, what is the probability of observing a stab cut given that the victim was 1) not stabbed with a single-edged blade, 2) stabbed with a different implement, or 3) slashed with a sharp implement? Again, logic and common sense - tempered with expertise - would indicate that these values would be very low. However, in the post-RvT climate, this is no longer a viable strategy. In order to evaluate this effectively, various relatively simple studies need to be developed where the probability of observing stab cuts (or damage that could be characterized as stab cuts) in alternative situations are developed. Having such data sets can then allow for the formation of clear and transparent probabilities that will lead to robust likelihood ratios that are firmly placed into the context of a case.

A recent initiative saw the launch of Research4Justice (www.research4justice.ac.uk), which is a repository for students' data sets conducted as part of their studies. This repository may allow for access to a viable data set to be used within an interpretational framework, thus satisfying the concerns expressed during the RvT judgment.

Another challenge that has to be highlighted in this review is the understanding of what constitutes "normal wear and tear", especially when it comes to underwear such as bras. It is clear that there are some assumptions being made that are easily and demonstrably incorrect. ${ }^{16}$ All clothing become worn and damaged through their lifetime, but there are many factors affecting this. Cigarette burn is a good example. If a person is a heavy smoker and heavy drinker, then cigarette burns on his/her clothing may be normal wear and tear. If, however, a person does not smoke, then cigarette burns become a lot more significant. One relevant example is a broken zipper, for example, on jeans. If the jean is old and obviously well worn, then a broken zipper may not necessarily indicate that the clothing was removed by force, whereas if the jean is reasonably new and in good condition, then the broken zipper may well indicate forceful removal. As seen with the bra with the hook and eye fastening, ${ }^{17}$ the question then becomes, what constitutes normal wear and tear over time.

Other than the study by Beever, ${ }^{18}$ there is very little work exploring this - at least in the context of forensic science.

\section{Summary}

There has been some excellent work conducted recently on improving aspects of textile damage analysis with efforts in projectiles analysis, decomposition, and understanding the interaction between the implement and the textile itself. There has also been excellent work exploring the phenomenon of falsified or deliberate textiles damage, which in turn raised an 
issue in that we do need a more fundamental understanding of normal wear and tear.

A lot of the findings relating to textiles damage appear to be a secondary consideration in the research project; for example, much of the research on projectiles was more about how the textiles affected the projectiles themselves, rather than an evaluation of the damage itself.

One of the things that became clear that just a little bit of research is subverting expectations, for example, Williams et al's study with respect to the hook and eye fastening, and that decomposition of buried remains appears to preserve the textile damage rather than exacerbating it.

It is clear that there is greater scope for further work in textiles damage, not only to develop a greater understanding of damage dynamics in sexual assaults but also with a view to chemical damage to clothing - especially in the context of increased numbers of the so-called acid attacks.

\section{Disclosure}

The author reports no conflicts of interest in this work.

\section{References}

1. Daroux FY, Carr DJ, Kieser J, Niven BE, Taylor MC. Effect of laundering on blunt force impact damage in fabrics. Forensic Sci Int. 2010;197(1-3):21-29.

2. Geisenberger D, Wuest F, Bielefeld L, et al. Fat from contused adipose tissue may cause yellow discoloration of clothes in blunt trauma victims. Forensic Sci Int. 2014;245:72-76.

3. Nic Daéid N, Cassidy M, McHugh S. An investigation into the correlation of knife damage in clothing and the lengths of skin wounds. Forensic Sci Int. 2008;179(2-3):107-110.

4. Kemp SE, Carr DJ, Kieser J, Niven BE, Taylor MC. Forensic evidence in apparel fabrics due to stab events. Forensic Sci Int. 2009;191(1-3): $86-96$.

5. Jussila J. Preparing ballistic gelatine-review and proposal for a standard method. Forensic Sci Int. 2004;141(2-3):91-98.

6. Carr DJ, Wainwright A. Variability of simulants used in recreating stab events. Forensic Sci Int. 2011;210(1-3):42-46.

7. Benson N, Oliveira dos Santos R, Griffiths K, et al. The development of a stabbing machine for forensic textile damage analysis. Forensic Sci Int. 2017;273:132-139.

8. Bostock EP, Williams G. Effect of decomposition on clothing damage evidence: A preliminary study. Crime Secur Soc. 2018;1(2).

9. Vennemann B, Dautel F, Braunwarth R, et al. Textile fibres along the bullet path-experimental study on a skin-gelatine composite model. Int $J$ Legal Med. 2008;122(3):213-218.
10. Wightman G, Wark K, Thomson J. The interaction between clothing and air weapon pellets. Forensic Sci Int. 2015;246:6-16.

11. MacPhee N, Savage A, Noton N, Beattie E, Milne L, Fraser J. A comparison of penetration and damage caused by different types of arrowheads on loose and tight fit clothing. Sci Justice. 2018;58(2):109-120.

12. Smyk D. Crossbow injuries: a case report. J Forensic Leg Med. 2009;16(6):343-345.

13. Opeskin K, Burke M. Suicide using multiple crossbow arrows. Am J Forensic Med Pathol. 1994;15(1):14-17.

14. Byard RW, Koszyca B, James R. Crossbow suicide: mechanisms of injury and neuropathologic findings. Am J Forensic Med Pathol. 1999;20(4):347-353.

15. Hinrichs R, Frank PRO, Vasconcellos MAZ. Short range shooting distance estimation using variable pressure SEM images of the surroundings of bullet holes in textiles. Forensic Sci Int. 2017;272:28-36.

16. Williams G, Haider I. Differentiating between genuine damage and falsified damage to a garment following an alleged sexual assault. $J$ Forensic Sci. 2012;57(6):1634-1636.

17. Daly DJ, Lee-Gorman MA, Ryan J. Distinguishing between damage to clothing as a result of normal wear and tear or as a result of deliberate damage: a sexual assault case study. J Forensic Sci. 2009;54(2):400-403.

18. Beever CE. Strengthening clothing damage analysis: quantification of stabbing force and weathering. 2014. Available from: eprints.hud. ac.uk/24700/1/Final_thesis_BEEVER.pdf. Accessed September 1, 2018.

19. Bostock E, Parkes G, Williams G. A novel method for the analysis of slash cuts to clothing. J Forensic Res. 2013;4:197.

20. Ueland M, Nizio KD, Forbes SL, Stuart BH. The interactive effect of the degradation of cotton clothing and decomposition fluid production associated with decaying remains. Forensic Sci Int. 2015;255:56-63.

21. Lowe AC, Beresford DV, Carter DO, et al. The effect of soil texture on the degradation of textiles associated with buried bodies. Forensic Sci Int. 2013;231(1-3):331-339.

22. Mitchell JL, Carr DJ, Niven BE, Harrison K, Girvan E. Physical and mechanical degradation of shirting fabrics in burial conditions. Forensic Sci Int. 2012;222(1-3):94-101.

23. Leung EH, Halliday DX. "Flashburning" - interpreting the presence of heat damage to a suspect's clothing and footwear in the investigation of fires. Sci Justice. 2010;50(4):187-191.

24. Taupin JM. Testing conflicting scenarios-a role for simulation experiments in damage analysis of clothing. J Forensic Sci. 1998;43(4):891-896.

25. Taupin JM. Clothing damage analysis and the phenomenon of the false sexual assault. J Forensic Sci. 2000;45(3):568-572.

26. Taupin JM. Arrow damage to textiles-analysis of clothing and bedding in two cases of crossbow deaths. J Forensic Sci. 1998;43(1):205-16207.

27. Taupin JM. Comparing the alleged weapon with damage to clothingthe value of multiple layers and fabrics. J Forensic Sci. 1999;44(1): 205-207.

28. Berger CE, Buckleton J, Champod C, Evett IW, Jackson G. Evidence evaluation: a response to the court of appeal judgment in R v T. $S c i$ Justice. 2011;51(2):43-49.

29. Cook R, Evett IW, Jackson G, Jones PJ, Lambert JA. A model for case assessment and interpretation. Sci Justice. 1998;38(3):151-156.

30. Boland CA, McDermott SD, Ryan J. Clothing damage analysis in alleged sexual assaults-the need for a systematic approach. Forensic Sci Int. 2007;167(2-3):110-115.
Research and Reports in Forensic Medical Science

\section{Publish your work in this journal}

Research and Reports in Forensic Medical Science is an international, peer-reviewed, open access journal publishing original research, reports, reviews and commentaries on all areas of forensic medical science. The manuscript management system is completely online and includes a very quick and fair peer-review system. Visit http://www.dovepress.com/ testimonials.php to read real quotes from published authors. 